\title{
LA PAZ TERRITORIAL EN LOS MONTES DE MARÍA: RETOS Y DESAFÍOS PARA SU CONSTRUCCIÓN
}

\author{
TERRITORIAL PEACE IN MONTES DE MARIA:
}

CHALLENGES FOR ITS CONSTRUCTION

Por: Amaranto Daniels Puello

omista de la Uni-

dad de Cartagena,

ialista en Planea-

para el Desarrollo

no-Rural. Magister

studios Políticos.

omicos de la
lad del Norte.

Recibido: 24 de marzo de 2015 - Aprobado: 29 de mayo de 2015

\section{RESUMEN}

La sociedad colombiana y la comunidad internacional se encuentran a la expectativa con los resultados de los diálogos entre el gobierno nacional y las Farc, que conduzcan a la terminación del conflicto armado en nuestro país. Este proceso se desarrolla en medio de un contexto político y social polarizado sobre la duración y viabilidad del mismo, una coyuntura económica marcada por la desaceleración de la actividad productiva nacional e internacional y una severa crisis institucional ante los recurrentes escándalos sobre corrupción pública-privada. Este artículo adelanta una reflexión sobre la puesta en marcha de los acuerdos que se suscriban entre las partes, específicamente la llamada paz territorial, su conceptualización, la identificación de los retos y desafíos que su desarrollo implica en aspectos como el modelo de convivencia a construir, las políticas públicas a desplegar, el rol de las organizaciones sociales, y los cambios en la arquitectura institucional que demanda esa apuesta hacia el postconflicto.

Palabras claves: Paz territorial, convivencia, conflicto, violencia, negociación.

\begin{abstract}
Colombian society and the international community are on the lookout with the results of the dialogue between the national government and the FARC, leading to the end of the armed conflict in our country. This process takes place in the midst of a polarized political and social context of the same duration and viability of an economic situation marked by the slowdown in the national and international productive activity and a severe institutional crisis to the recurrent scandals on public-private corruption. This article advances a reflection on the implementation of the agreements concluded between the parties, especially the so called territorial peace, its conceptualization, identification of the challenges that their development involves issues such as building a model of coexistence, deploy public policies, the role of social organizations, and changes in the institutional architecture that demand this commitment to the post-conflict.
\end{abstract}

Keywords: Territorial peace, coexistence, conflict, violence, trading.

\section{Antecedentes}

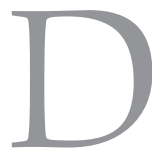

esde noviembre 19 de 2012 comienzan en La Habana ( Cuba) las negociaciones para la terminación del conflicto armado entre el gobierno nacional y las Fuerzas Armadas Revolucionarias de Colombia

Farc, -previamente se había definido una agenda, los procedimientos, países 
garantes y acompañantes-, teniendo como temas para las conversaciones los siguientes :

1. Política de desarrollo agrario integral

2. Participación política y garantías para la oposición.

3. El fin del conflicto.

4. Solución a las drogas ilícitas.

5. Atención a las víctimas.

Al examinar la agenda es innegable que los temas escogidos son las variables claves de un conflicto tan prolongado y degradado como el nuestro, el cual en las últimas tres décadas utiliza el territorio como núcleo para la presencia de los actores armados, y en su dinámica concentra en unas regiones ${ }^{1}$ la reproducción de la violencia asociada al conflicto. En ese sentido los temas de la agenda presentan manifestaciones o expresiones distintas en los territorios, por ejemplo el problema del narcotráfico es muy diferente en la Sierra Nevada de Santa Marta (zona productora), a los Montes de María (zona de tránsito y microtráfico), similar situación en lo relacionado con el tema de tierras, en donde en algunas regiones el problema es la concentración de la misma, y en otras es el conflicto por el uso del suelo para la preservación del medio ambiente. Resumiendo, tanto el gobierno como las Farc, desconocen que en esas regiones el ciclo de violencia reciente ha profundizado la heterogeneidad, el desequilibrio interno y la fragmentación espacial del territorio, más no la homogenización del conflicto que se pretende dar por concluido en las actuales conversaciones. He aquí una limitante que complejiza la puesta en marcha de los posibles acuerdos de la negociación en el futuro inmediato.

Una mirada retrospectiva sobre los avances de las negociaciones luego de 30 meses de iniciadas, nos revela entre otros resultados:

a) El logro de tres preacuerdos en los temas relacionados con el desarrollo rural, la participación política y las drogas ilícitas, a más de la existencia de 29 salvedades al respecto. Igualmente la creación de una Comisión de la Verdad, un plan de desminado conjunto y la posibilidad de un cese bilateral y definitivo de las hostilidades.

b) Actualmente se negocian temas cruciales como la atención a las víctimas y el fin del conflicto, cuya definición rebasa el ámbito nacional e incorpora la presencia de actores como la Corte Penal Internacional, alrededor de la disyuntiva entre el cumplimiento de los estándares de

En ese sentido reg como el Magdalen dio, el Oriente Anti ño, la Sierra Neva Santa Marta, el cor del Sumapaz, el S Tolima, los Mont María, la bota cau el Sur del país y el
del Catatumbo, otros. 
justicia transicional y la finalización de un conflicto que ha durado más de 50 años.

Con el propósito de dar a conocer el desarrollo de los diálogos la Oficina del Alto Comisionado ha recorrido el país, en especial aquellas áreas con mayor nivel de violencia y presencia de los actores del conflicto armado. Adicionalmente se reconoce por parte del Alto Comisionado para la Paz -Sergio Jaramillolos esfuerzos en direccionar una estrategia, en donde se plantea que la paz se construye desde las regiones, en lo que ha denominado la Paz Territorial, considerándola una apuesta esencial hacia el postconflicto, lo cual puede asumirse como una respuesta a la diversas dinámicas territoriales que asume la violencia y el conflicto en las últimas tres décadas, incluso a la carencia de una visión regional o territorial en la negociación

Ante el escenario descrito es de particular interés indagar si en los Montes de María de Bolívar y Sucre, en un territorio que posee una riqueza y complejidad -dada su diversidad ambiental y cultural- es posible la construcción de esa paz territorial, a partir del desarrollo del acuerdo para el fin del conflicto armado. Ahora bien, es válido explorar, si el acuerdo que se suscriba le da respuesta a los factores estructurales que origina la violencia asociada al conflicto armado en el territorio montemariano?. O si por el contrario es factible avanzar en una propuesta desde el territorio con las organizaciones sociales, quienes vienen elaborando una Agenda Territorial para la Convivencia y la Paz, desde su perspectiva y experiencia en las últimas tres décadas que incorpora los temas de La Habana, y a su vez contempla nuevos conflictos que no aparecen en la agenda referenciada.

\section{La paz territorial: Una aproximación a su conceptualidad}

Sincelejo en el o del Encuentro nstrucción de la

Territorial, orga-

Comisionado para

Comisionado para
$\mathrm{z}$ y al Fundación tes de María en 
la Paz Territorial significa poner en práctica en los territorios donde la violencia asociada al conflicto armado está presente en las últimas décadas, el acuerdo que se suscriba en La Habana. Agregando que el concepto de Paz Territorial (que no se ha elaborado), es una construcción que se debe hacer e incluye movilizar a la población de los territorios afectados por la violencia alrededor del tema de la paz. Complementa lo anterior la puesta en marcha de procesos de planeación participativa, de abajo hacia arriba, es decir que entre las autoridades locales y las comunidades identifiquen la problemática territorial para de manera concertada se formulen planes para transformar estas regiones, haciendo valer los derechos de la gente.

Finalmente, plantea que la Paz Territorial traza una nueva alianza basada en la lógica de la inclusión y la integración territorial, en donde la comunidad no sea receptora, si no, un actor fundamental de las políticas estatales. En otras palabras, se reitera el discurso que se viene impulsando desde las políticas públicas en los últimos años sobre la integración territorial y regional sin mayores logros. No sobra señalar, que el Comisionado reconoce los obstáculos que ha tenido ese proceso, como la continuación de la corrupción en la gestión administrativa, el clientelismo político, y la criminalidad organizada que expresan la ausencia de la soberanía del Estado en los territorios, para los cuales no precisa alternativas de respuesta.

Es indudable que los fundamentos sobre Paz Territorial son muy incipientes y muchos de ellos hace rato están en el ordenamiento constitucional, vigente ${ }^{3}$, los cuales después de muchos años de vigencia en la realidad no han generado cambios sustanciales para la ciudadanía y sociedad en general. Sin embargo es de reconocer que en su contenido es posible inferir la necesidad de avanzar en la construcción de paz, desde antes de finalizar el conflicto, como una acción de preparar el terreno para el despliegue de los acuerdos suscritos para evitar la recurrencia de la violencia y forjar las bases - a través del fortalecimiento de las instituciones- para remover las causas del conflicto.

Ahora bien, es pertinente precisar la complejidad que significa conceptualizar sobre la construcción de paz. Desde los estudios de J. Galtung $(1976,2003)$, la propuesta de Agenda para la Paz (ONU 1992) y R. Grasa (2010) es evidente las distintas posiciones sobre su elaboración, contextualización y el campo del conocimiento en donde se ubica, pero coinciden en la importancia de la naturaleza del conflicto, su transformación creativa, la interdisciplinariedad y la respuesta a las causas estructurales que los originan. Por su parte A. Retberg (2003), traza una línea desde la Agenda para la Paz de Naciones 
Unidas, asumiéndola como un concepto multidimensional e impreciso, ya que sus interpretaciones van desde una posición como la superación de las secuelas específicas del conflicto en una visión minimalista, pasando por responder a las causas estructurales del conflicto (visión maximalista), hasta una perspectiva intermedia en la cual se avanza de la emergencia humanitaria a la ayuda para el largo plazo.

Recapitulando no hay una elaboración conceptual sobre Paz Territorial y la apuesta de la Oficina del Comisionado es la de evolucionar en el marco de normas y leyes que promueven el Estado Social de Derecho. Es claro entonces la necesidad de desarrollar e instrumentar la construcción de paz como una respuesta integral a los factores estructurales que motivan el conflicto armado y la violencia en Montes de María. En el marco de esas deliberaciones surge la oportunidad de concretar ese propósito desde un proceso de convivencia en la subregión, no como un concepto opuesto a lo de Paz Territorial, si no como la base y/o referente que se construye a partir de la realidad del territorio.

En este aspecto es clave establecer como concebimos la convivencia?, desde su etimología la convivencia es entendida como vivir con otro(s) o junto a otro(s), más no simplemente al lado del otro (A. Gómez Müller 2008). Parafraseando a Gómez Müller, en nuestro país a lo largo de la historia, se ha incorporado la convivencia ciudadana en términos de libertad, igualdad y fraternidad, o sea desde su fundamento ideológico republicano se hace un reconocimiento del otro, pero en la realidad estos principios no se cumplen ante la inoperancia de las instituciones y las actitudes políticas excluyentes.

Dese ese punto de vista son indiscutibles los retos que implica alcanzar la convivencia en un territorio como los Montes de María, en donde las características multiculturales y étnicas (presencia de afros, indígenas, campesinos), de los actores sociales (líderes comunitarios, docentes, mujeres, jóvenes, victimas y desmovilizados), amerita sopesar de qué manera es factible convivir en ese espacio en donde la población posee costumbres y valores distintos, e igualmente intereses económicos diversos. Al respecto véase el mapa adjunto (figura 1) el cual muestra los múltiples intereses económicos, sociales, culturales y ambientales del territorio montemariano; donde sobresale la disputa en el territorio por la puesta en marcha de una zona de reserva campesina, la titulación de áreas colectivas a comunidades afros en Paraíso y San Cristóbal (San Jacinto) y la presencia de vastas áreas de explotación minera en el territorio colindantes con zonas de reserva naturales. 

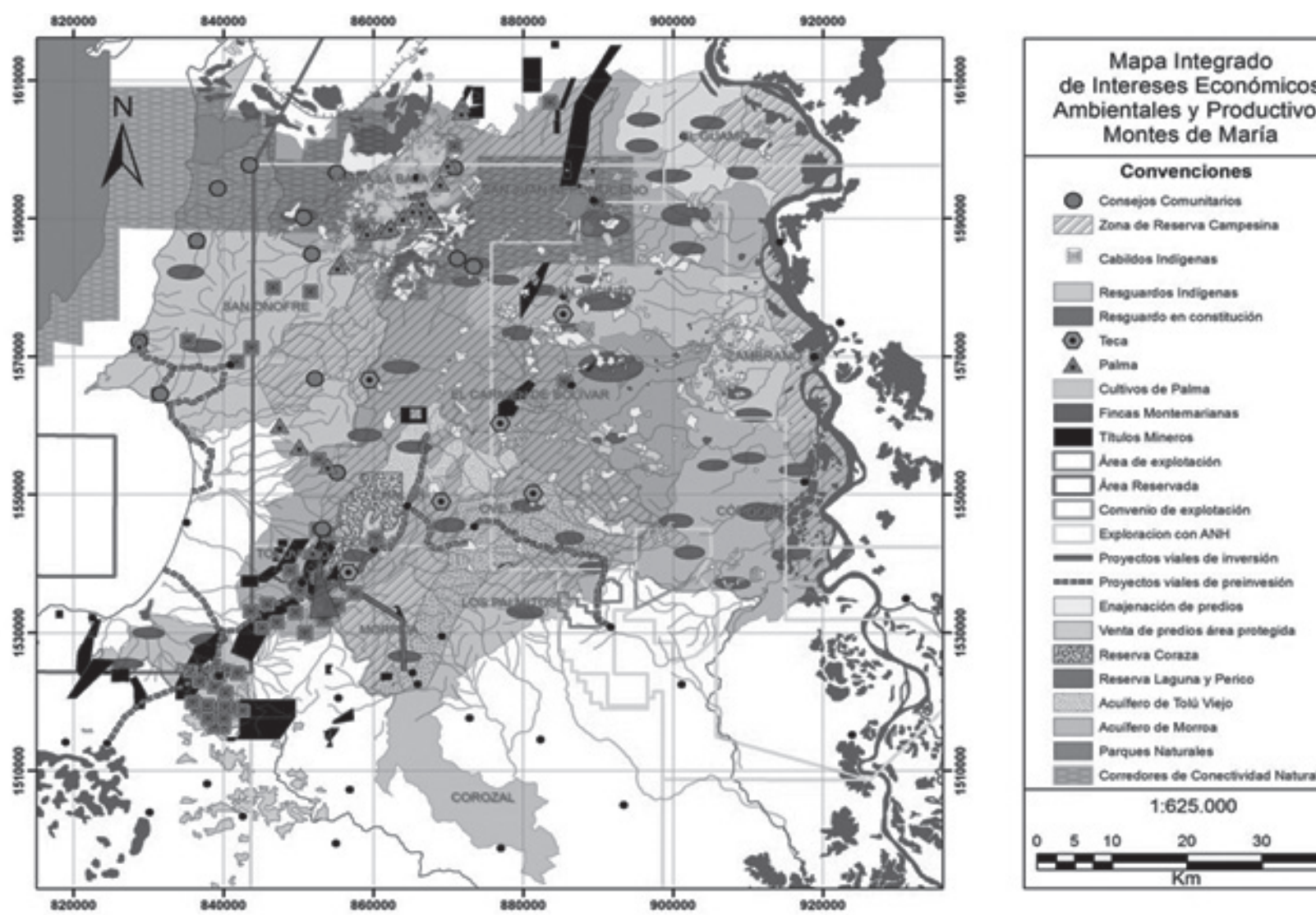

Figura 1. Montes de María. Territorio, población y estructura productiva-econór

A partir de la reflexión anterior y teniendo claridad de las limitantes existentes es muy legítimo apostarle a la construcción de la convivencia, como una relación entre los seres humanos que facilita el despliegue de la libertad individual, la existencia de unos espacios libres para el desarrollo de su proyecto de vida personal, y la generación de condiciones y oportunidades que hagan viable la participación de todos en la organización de la vida en comunidad (J. García R. 2007). Para el caso que nos ocupa la convivencia territorial surge desde personas capaces de compartir un espacio común, de relaciones, manteniendo su propia identidad cultural y política como tal; a la vez que ese grupo de personas comparten un conjunto de normas, valores morales y de creencias, que conforman su cultura, teniendo como principios rectores el respeto a la diferencia, la libertad, la justicia, la igualdad y la cooperación entre otros.

La materialización de ese propósito implica reconstituir un conjunto de procedimientos desde la educación, como sustento de una socialización para la vida en comunidad, a más de ocuparse de la forma en que los ciudadanos asumen el ejercicio de los derechos y sus responsabilidades para transformar 
las instituciones que nos regulan. Luego entonces esa convivencia, está apuntalada por unos valores compartidos - desde los derechos humanos- y unos ejes direccionantes como: la tolerancia, la participación, la autonomía y la responsabilidad para con nuestros conciudadanos. Resumiendo, este es el primer reto para la convivencia territorial, el cual implica una innovación radical del sistema educativo montemariano como sustento de ese nuevo orden que se pretende construir.

Esa fundamentación de la convivencia, se asume desde la mirada de Aristóteles quién caracterizó al hombre como un animal político, racional (A. Cortina, 2001) que permite ir delineando los pasos para romper con lo que tradicionalmente ha pasado en los Montes de María y en otras partes del país, y es el enfoque de la convivencia como dominación (J. García R. 2007), en donde algunos tienen la potestad para decidir según su voluntad y el resto están sometidos a ese poder. Ese tipo de convivencia propicia, que en un momento dado una parte de la población no se siente reconocida generándose nuevos ciclos de violencia, evidenciando una sociedad en donde no hay confianza, ni procesos que la garanticen.

\section{Bases para la convivencia territorial}

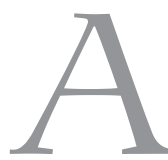

ntes de describir las fases para estructurar la convivencia es oportuno de manera sucinta, mostrar las visiones que tienen los con base en la participación durante los dos últimos años en los múltiples seminarios, diplomados y talleres realizados en el territorio al respecto.

Para la mayoría de los montemarianos hay un desconocimiento de las conversaciones entre el gobierno colombiano y las Farc, lo cual va desde el acuerdo general pasando por lo suscrito en los puntos sobre desarrollo rural, participación política y drogas ilícitas, hasta apreciar los cambios que este proceso tendría hacia el futuro inmediato sobre el territorio y las comunidades, en términos de bienestar, seguridad y convivencia.

Los miembros de las organizaciones sociales y populares que acceden a espacios de difusión y/o análisis sobre los diálogos, poseen una perspectiva crítica sobre la utilidad de los mismos y la posibilidad de cambiar las condiciones de vida en el territorio, ante la bajísima credibilidad en el Estado y sus instituciones para la efectividad de las políticas públicas o programas. En ese sentido traen a colación los intentos de reforma agraria o la atención a las víctimas del 
conflicto armado, y la constante corrupción político-administrativa como eje de la relación Estado-sociedad civil.

De igual manera hay suficiente certeza que la firma del acuerdo entre las partes no significa la cesación de los conflictos sociales, políticos, ambientales y económicos existentes en la subregión. Por ejemplo: La aparición de conflictos ambientales ante la presencia de exploraciones de hidrocarburos (ANH 2008) y la explotación de gravas, minerales y calizas (véase anexos) ; asimismo la política agropecuaria que privilegia el monocultivo de la palma de aceite en detrimento de la economía campesina, y el acceso al uso del agua del distrito de riego en Marialabaja entre los productores corroboran esa afirmación.

El punto de partida para la convivencia en el territorio es examinar las características del ordenamiento social existente, cuál es la sociedad que tenemos? En ese sentido, los Montes de María tiene como rasgos esenciales en su orden social la presencia de elementos tradicionales como el talante señorial o aristocrático de una sociedad terrateniente; la fragilidad del estado de derecho y una incipiente ciudadanía activa e instituciones políticas precarias. A ello se agrega la ausencia de reglas de juego como medios de regulación e interacción al interior de su entramado social y de una presencia diferenciada del Estado, es decir un funcionamiento de las instituciones locales por fuera de los diseños y marcos jurídicos vigente, además de la manera como se articulan en lo político, económico y cultural con el conjunto de la nación (González, Bolívar y Vásquez. 2003).

En otras palabras, estamos en presencia de un proceso de modernización muy particular y complejo a la vez, cuyas particularidades son muy diferentes al modelo occidental ${ }^{4}$ (J. Beriain, 2005). Es decir un proceso asimilado a un agregado de notas provisorias que comportan algunas condiciones mínimas que pueden ser exigidas de las instituciones sociales allí presentes (J. Beriain, 2005). Parafraseando a Beriain, ese conjunto de notas provisorias intenta explicar la continuidad de una ambivalencia en donde coexisten tanto la violencia como intentos de orden y/o convivencia durante su reconstrucción o redefinición, y las decisiones colectivas muestran contradicciones entre el orden que se diseña para su funcionamiento y el comportamiento de los grupos sociales ante la compleja realidad territorial, creando una mayor incertidumbre, rupturas y desorden a su interior (Z. Baumant, 2005).

En segundo lugar es indispensable avanzar hacia la reconciliación al interior del territorio luego del ciclo reciente de violencia para lo cual se requiere entre ${ }^{4}$ En la modernida
ropea el Estado-N
posee el monopo
la fuerza militar,
dadano asume 1
beranía como base
sociedad civil al in
de la esfera públi
donde las institu
regulan la intera
entre los ciudada
el Estado. 
otros aspectos: Superar el déficit de un mínimo de verdad desde la visión de las víctimas, en el sentido de conocer o saber todo lo que sucedió antes que interpretar o juzgar lo sucedido (A. Gómez Muller, 2008); es decir una verdad real o desnuda a través de relatos o testimonios para luego saber lo que ha pasado, o sea se requiere conocer por qué ha sucedido lo acontecido. En este punto las víctimas (a partir de la verdad real) indagan por una verdad explicativa o una verdad sentido como la define Gómez Muller, que permita interpretar y evaluar esos hechos a la luz del contexto del conflicto armado y de las normas nacionales e internacionales que los regulan.

Desafortunadamente la ley 975/05 -verdad, justicia, reparación y reconciliación- no logra ese grado de desarrollo, ya que a pesar de las treinta y siete declaraciones de los miembros del bloque paramilitar Héroes de los Montes de María y los testimonios de las víctimas, no sabemos por qué pasó este ciclo de violencia ${ }^{5}$, dando como resultado una verdad fragmentada y relativizada que no promueve el acceso a la Justicia y a la Reconciliación al interior de la sociedad. En este sentido es de vital importancia la creación de una Comisión de la Verdad en Montes de María ${ }^{6}$, de carácter pluralista en su conformación, que permita darle respuesta a inquietudes como: a) Los orígenes, dinámica y duración del conflicto armado en el territorio; b) Establecer el rol de los denominados no combatientes, de los financiadores y soportes financieros de los actores armados ilegales, c) Identificar los lugares de las víctimas y desaparecidos, d) El rol del Estado en cuanto a la omisión u acciones que en su momento favorecieron las actividades que afectarán la seguridad y los derechos humanos en el territorio.

En cuanto a la reconciliación que se propone, es posible concebirla en términos de ser un proceso con sus propios ritmos, acorde al contexto, de carácter estructural -implica cambios en las instituciones, la cultura-, que pretenda restaurar y sanar heridas y que en últimas procure afianzar procesos democráticos en situaciones de postconflicto. Complementa esta fase la generación de capacidades para resolver los conflictos en el territorio lo cual requiere entre otros atributos:

a) Fortalecer la capacidad (a partir del hacer - poder) para que los actores sociales montemarianos construyan y desarrollen procesos de diálogo, cooperación y mediación desde una visión política de carácter colectivo que permita establecer una ruta de acciones que haga viable resolver las diferencias de una manera incluyente. 
b) Establecer unas redes de apoyo en esa perspectiva de resolución incluyente de los conflictos apoyado en la generación de confianza y de evitar la polarización con miras a un trabajo conjunto y articulado. En el territorio, la participación en escenarios como las mesas de trabajo de campesinos, afros, jóvenes entre otros ha permitido ir edificando unas bases para generar capacidades en el manejo de los conflictos.

En tercer lugar la participación política-electoral debe ir más allá del concepto de participación, el cual es reduccionista frente a las reformas esenciales como las que implican los desarrollos de la agenda de La Habana. Es imperativo acometer políticas y programas que posibiliten la construcción de una cultura política territorial -lo cual implica cambios estructurales en el sistema educativo-, así como la adopción de normas en lo atinente a las dinámicas de la participación política y la democracia local.

Para esa intencionalidad es la escuela entendida como el centro generador de conocimientos para la comunidad en general, soportado en un modelo pedagógico territorial en donde la estructura curricular es fruto de una elaboración colectiva, adaptable al contexto, con un enfoque didáctico flexible, que facilita tener en cuenta las diferencias de origen -político, social, cultural y étnicas-, y en donde el contenido privilegie la diversidad política-ideológica, el manejo de los conflictos y la convivencia desde la argumentación dialógica y razonable.

Resumiendo es necesario superar la visión instrumental y reduccionista que subyace en las competencias ciudadanas ${ }^{7}$, la cual normaliza los comportamientos del ciudadano ante el énfasis centrado en el desempeño, lo cual finalmente tiene efectos sobre la autonomía del individuo. Adicionalmente es muy importante reconocer y entender como es la formación de la identidad política al interior de la sociedad montemariana; es decir, como se constituye esa identidad, desde lo personal, desde lo colectivo, desde lo territorial, o desde su habitus como lo define Bourdieu ${ }^{8}$. Sumado al rol de la escuela, es de vital interés impulsar procesos desde la educación popular, a partir de su discurso político y pedagógico posibilitando una intervención concreta y transformadora de la participación política en Montes de María. Como bien lo plantea Marco R Mejía El acto educativo de la educación popular es fundamentalmente un acto de transformación del individuo, de los grupos y de las organizaciones sociales, y serían procesos ficticios si el conflicto no hiciera presencia (2002). 
Cierra esta parte, la perentoria necesidad de una profunda reforma al régimen de partidos políticos que incluyan responsabilidades y sanciones a las colectividades por actuaciones propias y de sus miembros, las cuales deben ir más allá del abandono y pérdida de la curul; igualmente está lo relacionado con la financiación de las campañas políticas, el estatuto de la oposición y el acceso a los medios de comunicación en condiciones de equidad. Sintetizando, la construcción de una cultura política territorial se asume como un proceso en múltiples lugares de la comunidad desde el hogar, la escuela que trascienda al contexto, generando prácticas y reglas que ayuden a modelar una identidad social e individual en el comportamiento político del ciudadano montemariano y su relación público-privado con el Estado en sus diversos niveles.

En cuarto lugar, es definitivo abrir el debate sobre la institucionalidad ${ }^{9}$ que requiere la puesta en marcha de los acuerdos de La Habana a nivel regional, es innegable que los esfuerzos acometidos por el Estado en materia de descentralización política-administrativa -en Montes de María- no ha logrado aún el reto de transformar la cultura y prácticas organizacionales de la estructura pública en el territorio. En la realidad es un modelo de gestión descentralizada, pero controlada desde el nivel central en su orientación -definición del gasto público y las prioridades del mismo- en donde los actores responsables (Alcaldes - Gobernadores) es posible asimilarlos a unos agentes regidores (Jolly, 2007) en el gobierno del territorio, con el agravante de la perseverancia del clientelismo y la corrupción político-administrativa ${ }^{10}$ como ejes de la gestión pública montemariana.

Ese tipo de gestión, se refleja en el proyecto de ley que cursa en el Congreso de la República para la aprobación del plan nacional de desarrollo 2014-2018 "Todos por un nuevo país. Paz, Equidad, Educación”, el cual en sus objetivos le apunta a fortalecer condiciones del proceso de construcción de paz y garantizar su sostenibilidad, sin embargo su direccionamiento estratégico se centraliza en el nivel nacional obviando el rol de las regiones, demostrando severas contradicciones con el discurso de la "Paz Territorial", tal como se muestra a continuación:

a) Los artículos 97, 102 entre otros introduce cambios en la normatividad vigente-mediante facultades al presidente por seis meses -en materia de desarrollo rural, la política de tierras- incluyendo la reducción o supresión de instituciones -, así como lo atinente a la política de créditos agropecuarios y el manejo de los baldíos, modificando la ley 160 de 1994, específicamente el artículo 76. 
b) El artículo 123, crea el Consejo Interinstitucional del Postconflicto ${ }^{11}$, como órgano encargado de la coordinación interinstitucional, entre los niveles nacional y territorial, para facilitar la toma de decisiones que articulen el alistamiento e implementación de los acuerdos que se deriven de las conversaciones entre el Gobierno Nacional y las organizaciones armadas ilegales.

En ese aspecto los cambios en el modelo descentralizador en la perspectiva de implementar los acuerdos de La Habana, requieren cambios tales como: la funcionalidad de espacios de coordinación y correspondencia entre la oferta nacional (recursos humanos, técnicos, financieros e institucionales) frente a las demandas territoriales (provisión), con miras a lograr un equilibrio que haga viable el desarrollo de los acuerdos y el afianzamiento del gobierno territorial. De otra parte garantizar la capacidad para lograr mantener el control y la autoridad gubernamental en el territorio, como respuesta a esa tradición de Estado endémico, en donde aún existe una lucha por el control territorial (presencia de bandas criminales emergentes), aun cuando en menor proporción a la de hace dos décadas.

En síntesis es prioritario, los ajustes al modelo de descentralización para gobernar el territorio, soportado en la re-construcción de una capacidad gubernamental para facilitar que las políticas, programas y proyectos se desplieguen en el territorio a través de las acciones y los recursos de las organizaciones o actores que gobiernan para lograr el impacto que se pretende en los acuerdos suscritos entre el gobierno colombiano y las Farc.

Para ello se plantea la creación de un Consejo Territorial para la Convivencia y la Paz, cuya funciones claves serían entre otras: a) Ser el ente responsable, coordinador, y supervisor de la puesta en marcha de los acuerdos suscritos con las Farc y demás organizaciones subversivas, b) En su conformación estaría integrado por representantes del gobierno nacional, los entes territoriales, las organizaciones sociales y el empresariado presente en la subregión, c) Asumir el direccionamiento estratégico para la formulación, ejecución y seguimiento a los recursos de los distintos planes/proyectos, en materia de desarrollo, rural, participación política, reintegración y drogas ilícitas, durante los próximos diez años o la temporalidad definida para el desarrollo de acuerdos suscritos en La Habana.

En últimas, es definitiva la presencia y participación de representantes de las organizaciones sociales montemarianas en la toma de decisiones sobre las políticas o programas para materializar los acuerdos entre el gobierno y las 
Farc, como un mecanismo idóneo para posibilitar que la corrupción políticoadministrativa no incida de manera restrictiva sobre las metas de las políticas públicas, y continúe la depredación de los recursos públicos.

\section{A manera de conclusiones...}

T a construcción de la paz territorial en Montes de María se concreta desde la consolidación de la convivencia y la correspondencia para una Agenda regional, en consonancia con la paz nacional, cuya base sea la confianza entre los actores sociales participantes para viabilizar la reintegración, la reconciliación y la armonía, al interior de la sociedad colombiana.

La agenda territorial para la paz se instrumentaliza con un Pacto por la Convivencia en donde los diferentes actores (gobierno, organizaciones, desmovilizados e instituciones), se constituyen en facilitadores que legitiman los acuerdos políticos, a más de propiciar que la agenda se convierta en política pública territorial para que su desarrollo posibilite superar las causas estructurales del conflicto armado en los Montes de María. Complementa este cometido la generación de una infraestructura que permita el trámite incluyente de los conflictos territoriales, desde la mediación, la conciliación y la transformación de esas diferencias, aprovechando la experiencia de las organizaciones sociales y populares al respecto. Es decir pasar de las conversaciones entre las élites políticas o empresariales a la variedad de actores sociales -indígenas, afros, campesinos y mujeres entre otros- que hacen presencia en la subregión montemariana.

El mayor desafío de los Montes de María en la perspectiva de construir la convivencia y la concordia, se ubica en la creación de las capacidades institucionales para el cumplimiento de los acuerdos en el territorio, lo cual supone la modificación del orden social construido. De allí la importancia de la gestión del conocimiento -particularmente la educación y la academia- como fundamento para evitar repetir el incumplimiento de los acuerdos y romper con esa tradición de fracasos en las iniciativas y políticas públicas. A ello se agrega la necesidad de participación de la comunidad montemariana, desde la toma de decisiones hasta el ejercicio de la veeduría pública al Pacto por la Convivencia, para efectos de verificar su nivel de cumplimiento.

Finalmente, es significativo consolidar los procesos de participación ciudadana de las organizaciones sociales montemarianas, específicamente alrededor de su presencia en la formulación de las políticas públicas territoriales (entendidas como los medios a través de los cuales el Estado Social de Derechos se materializa y deja de ser retórica); el ejercicio del poder territorial ( el abordaje 
del poder político local desde la emergencia de los actores políticos sociales y comunitarios); y la rendición de cuentas o auditoría social (eficiencia, calidad del gasto y transparencia desde afuera por la ciudadanía), durante la vigencia de los acuerdos logrados para la finalización del conflicto armado.

\section{BIBLIOGRAFÍA}

MORENO DURÁN, A. \& RAMíREZ J. (2013). Pierre Bourdieu. Proyección siglo XXI. Instituto Latinoamericano de Altos Estudios. ISBN 978-958-8492-25-4. Páginas 30-46

BAUMAN ZYMUNT. (2005). Modernidad y ambivalencia. ISBN 84-7658-708-2. Páginas 19-59. Editorial Antrophos.

BERIAIN, Josetxo. (2005) Modernidades en disputa. ISBN 84-7658-722-8. Páginas 11-75. Editorial Antrophos.

CORTINA, Adela. (2001). Ciudadanía política: Del hombre político al hombre legal. www.c.s.usb. $\mathrm{ve} /$ sites/

DANielS PUEllo, Amaranto \& Múnera CAVAdía, Alfonso. (2011). Los Montes de María. Región conflicto y desarrollo productivo. ISBN 978-958-9230-63-3. Instituto Internacional de Estudios del Caribe-Universidad de Cartagena. Editorial Antropos.

GALTUNG J. (2003). Paz por medios pacíficos. Paz y conflicto, desarrollo y civilización. (T. Toda, Trad.) Bilbao: Gernika Gogoratuz.

GARCÍA, J. (2007). Educación para la ciudadanía. Barcelona: Espiral. Consultado en http://www. fespinal.com/espinal/llib/es149.pdf

GONZÁLEZ, Fernán; BOLÍVAR, Ingrid \& VARGAS, Teófilo. Violencia política en Colombia. De la nación fragmentada a la construcción del estado. Cinep. 2003 paginas 218-232

GÓMEZ MULLER, Alfredo. La reconstrucción de Colombia escritos políticos. La Carreta EditoresPlaneta Paz. ISBN 978-958-9839-5-7. Marzo 2008. Medellín. Colombia. Páginas 8-45.

GRASA, R. (2010). Cincuenta años de evolución de la investigación para la paz. Tendencias y propuestas para observar, investigar y actuar. Barcelona: Generalidad de Catalunya.

ICFES Pre Saber 11, 2012 en wwww.icfes.gov.co

ICFES Pre Saber 5-9, Pensamiento ciudadano. wwww.icfes.gov.co

ICFES (2012). Resultados/reportes saber 359. wwww.icfes.gov.co

MEJÍA MARCO, Raúl. La educación popular. Una experiencia de saber pedagógico. La memoria activa del saber pedagógico en la contemporaneidad. Universidad de Antioquia. Mimeo. 2002.

No. 15. Agosto de 2015 Palobra 
Observatorio de Cultura Política, Paz, Convivencia y Desarrollo de los Montes de María (2010). Los Montes de María: Entre la incertidumbre y la esperanza. Informe de Coyuntura 2009. Instituto Internacional de Estudios del Caribe. Universidad de Cartagena, www.sitmma.org.

Observatorio de Cultura Política, Paz, Convivencia y Desarrollo de los Montes de María (2011). Los Montes de María: La gente se atreve a soñar otra vez. Informe de Coyuntura 2010. Instituto Internacional de Estudios del Caribe. Universidad de Cartagena, www.sitmma.org.

Observatorio de Cultura Política, Paz, Convivencia y Desarrollo de los Montes de María Sistema de Información Territorial (2011). Encuesta de caracterización de los Montes de María. Línea Base. Instituto Internacional de Estudios del Caribe. Universidad de Cartagena, www. sitmma.org.

Proyecto de Ley. Cámara de Representantes. Por la cual se expide el Plan Nacional de Desarrollo 2014 -2018. "Todos por un nuevo país" DNP. Febrero 2015.Bogota.

Territorialidad y sectorialidad de las políticas públicas. Aspectos analíticos y utilización normativa de un esquema de análisis de las políticas públicas en el territorio. Jean François Jolly en: Ensayos sobre Políticas Públicas. Universidad Externado de Colombia. 2007 ISBN 978-958710-305-2 


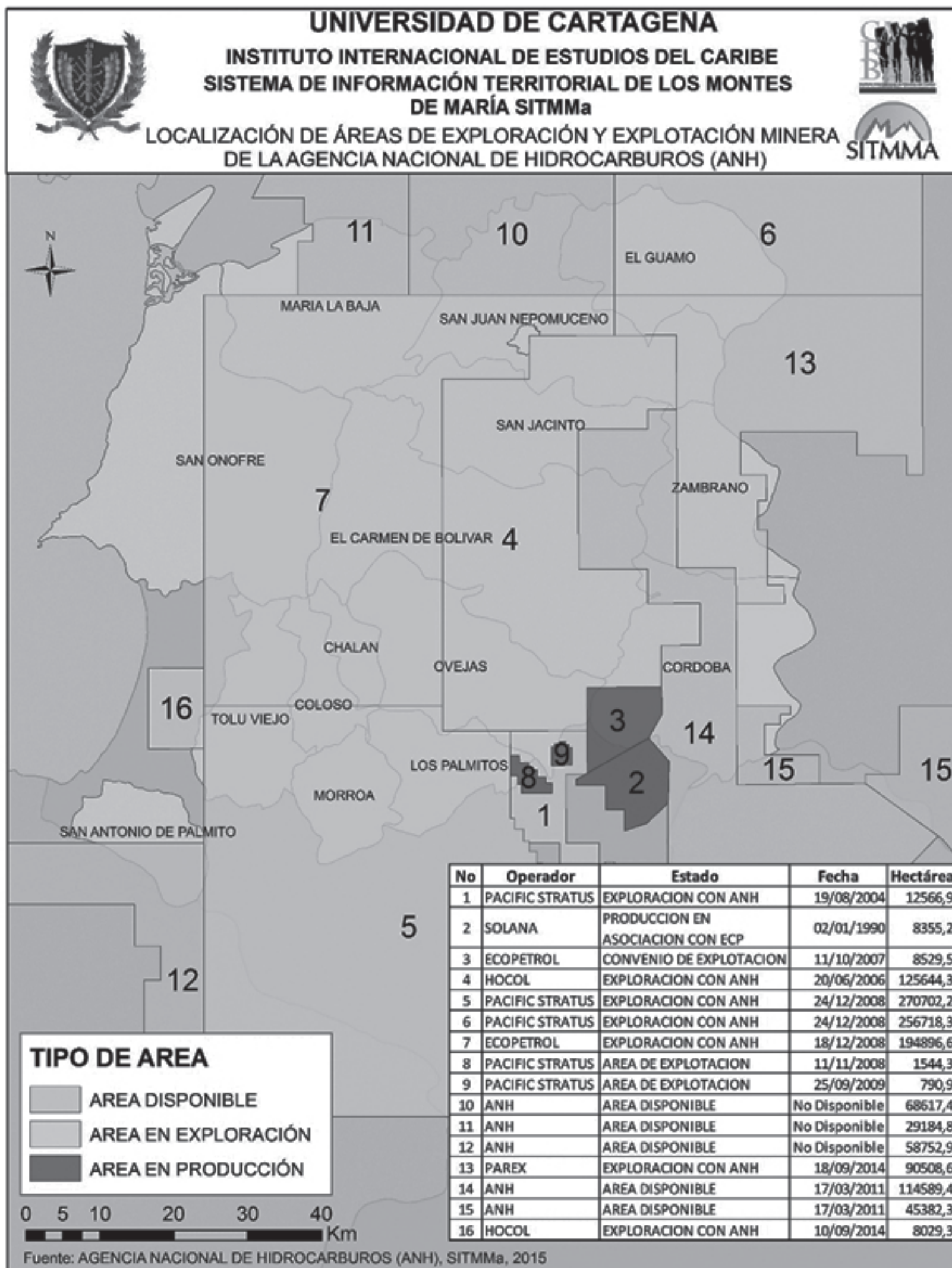

http.//www.sitmma.o 


\section{UNIVERSIDAD DE CARTAGENA INSTITUTO INTERNACIONAL DE ESTUDIOS DEL CARIBE SISTEMA DE INFORMACIÓN TERRITORIAL DE LOS MONTES DE MARIA SITMMa ZONAS MINERAS}
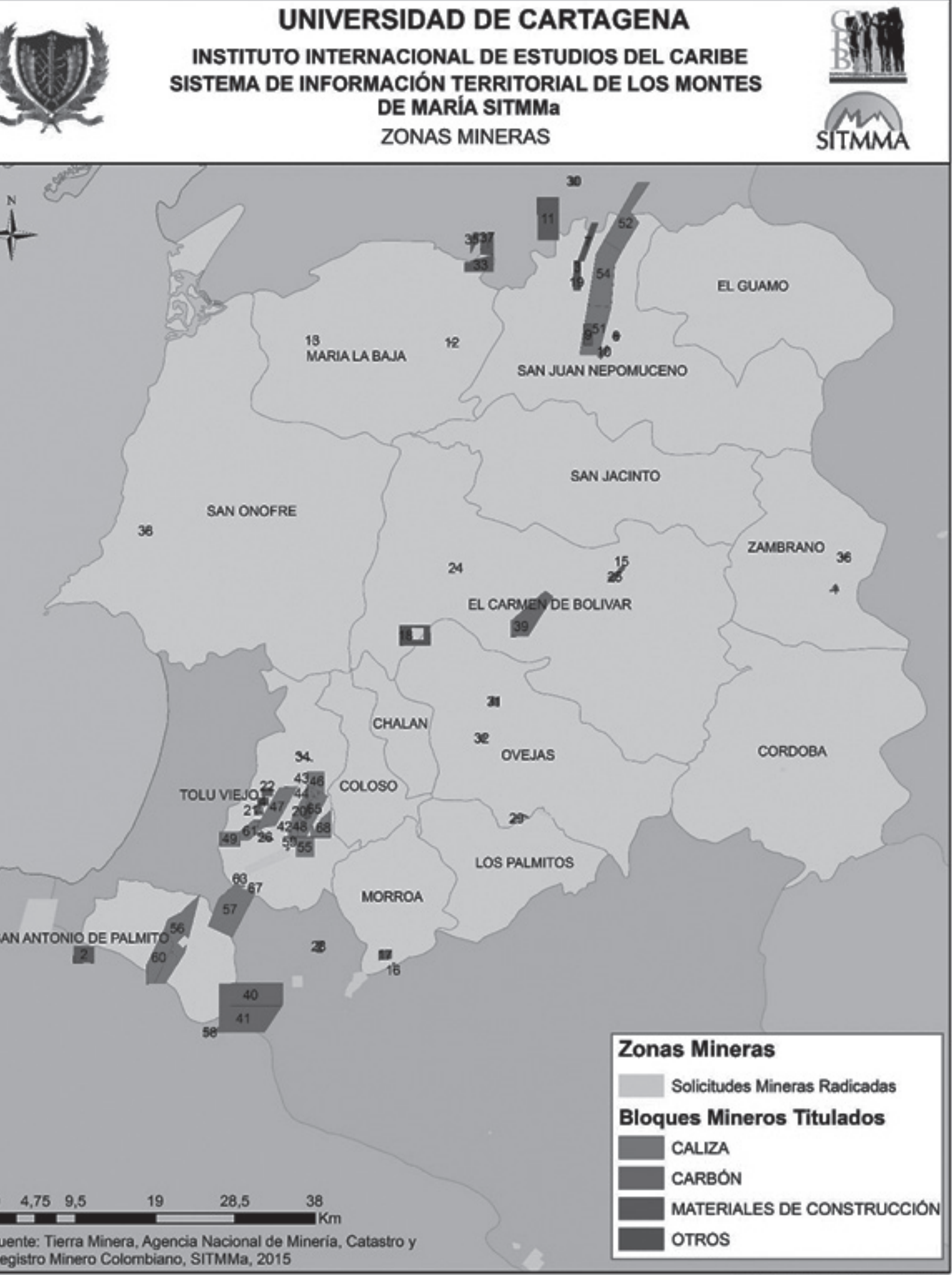

http.//www.sitmma.org 


\section{BLOQUES MINEROS TITULADOS}

\begin{tabular}{|c|c|c|c|c|c|}
\hline No & FECHA & MINERALES & CLASIFICACIÓN & TITULARES & HECTARE \\
\hline 1 & $30 / 07 / 1997$ & GRAVA, ARENA & OTROS & $\begin{array}{l}\text { (8600785657) MONTERREY } \\
\text { FORESTAL LTDA }\end{array}$ & 38,63 \\
\hline 2 & $25 / 08 / 2006$ & $\begin{array}{c}\text { DEMÁS CONCESIBLES, MATERIALES } \\
\text { DE CONSTRUCCIÓN, CALIZA }\end{array}$ & $\begin{array}{l}\text { MATERIALES DE } \\
\text { CONSTRUCCIÓN }\end{array}$ & $\begin{array}{l}\text { (9001535833) COMPAÑÍA } \\
\text { MINERA TANURIN S.A }\end{array}$ & 470,97 \\
\hline 3 & $02 / 12 / 1991$ & ARCILLA, SILICEOS & OTROS & $\begin{array}{c}\text { (8901002510) CEMENTOS } \\
\text { ARGOS S.A }\end{array}$ & 99,00 \\
\hline 4 & $11 / 08 / 1993$ & CHERT, DEMÁS CONCESIBLES & OTROS & $\begin{array}{c}\text { (8901002510) CEMENTOS } \\
\text { ARGOS S.A }\end{array}$ & 109,92 \\
\hline 5 & $31 / 05 / 2007$ & $\begin{array}{l}\text { DEMÁS CONCESIBLES, } \\
\text { MATERIALES DE CONSTRUCCIÓN }\end{array}$ & $\begin{array}{l}\text { MATERIALES DE } \\
\text { CONSTRUCCIÓN }\end{array}$ & $\begin{array}{l}\text { (22953925) ALBERTINA } \\
\text { TORRES ESCAMILLA }\end{array}$ & 75,77 \\
\hline 6 & $03 / 10 / 2008$ & $\begin{array}{l}\text { DEMÁS CONCESIBLES, } \\
\text { MATERIALES DE CONSTRUCCIÓN }\end{array}$ & $\begin{array}{l}\text { MATERIALES DE } \\
\text { CONSTRUCCIÓN }\end{array}$ & $\begin{array}{c}\text { (92275274) PABLO } \\
\text { ALEJANDRO ÁLVAREZ ALVIZ }\end{array}$ & 8,39 \\
\hline 7 & $23 / 02 / 2007$ & CHERT, DEMÁS CONCESIBLES, ARCILLA & OTROS & $\begin{array}{c}\text { (8901002510) CEMENTOS } \\
\text { ARGOS S.A }\end{array}$ & 330,30 \\
\hline 8 & $29 / 04 / 2008$ & $\begin{array}{l}\text { AGREGADOS PARA CONSTRUCCIÓN, } \\
\text { ASFALTO, RECEBO, GRAVA, ARENA, } \\
\text { CONGLOMERADO, ARENISCAS }\end{array}$ & $\begin{array}{l}\text { MATERIALES DE } \\
\text { CONSTRUCCIÓN }\end{array}$ & $\begin{array}{l}\text { (73226076) FERNANDO DE } \\
\text { JESÚS DE ORO LORA }\end{array}$ & 50,64 \\
\hline 9 & $29 / 08 / 2007$ & MATERIALES DE CONSTRUCCIÓN & $\begin{array}{l}\text { MATERIALES DE } \\
\text { CONSTRUCCIÓN }\end{array}$ & $\begin{array}{c}\text { (73351320) DAX GILBERT } \\
\text { GUILLEN BIOJO, (7931359) } \\
\text { CHARLTON ADOLFO } \\
\text { LOBELO PUELLO }\end{array}$ & 250,00 \\
\hline 10 & $29 / 08 / 2007$ & $\begin{array}{l}\text { MATERIALES DE CONSTRUCCIÓN, } \\
\text { ARCILLA, CALIZA, ZAHORRA }\end{array}$ & $\begin{array}{l}\text { MATERIALES DE } \\
\text { CONSTRUCCIÓN }\end{array}$ & $\begin{array}{l}\text { (8237502) PEDRO MANUEL } \\
\text { OSORIO ARRIETA }\end{array}$ & 85,15 \\
\hline 11 & $07 / 10 / 2008$ & MATERIALES DE CONSTRUCCIÓN & $\begin{array}{l}\text { MATERIALES DE } \\
\text { CONSTRUCCIÓN }\end{array}$ & $\begin{array}{l}\text { (8600007624) LADRILLERA } \\
\text { SANTAFE S.A. }\end{array}$ & $1.249,8 \varepsilon$ \\
\hline 12 & $15 / 05 / 2008$ & ASOCIADOS, GRAVA, ZAHORRA & OTROS & $\begin{array}{l}\text { (30840406) LADY } \\
\text { CORREA BARRIOS }\end{array}$ & 12,60 \\
\hline 13 & $15 / 05 / 2008$ & $\begin{array}{l}\text { DEMÁS CONCESIBLES, GRAVA, } \\
\text { ARENA, ZAHORRA }\end{array}$ & OTROS & $\begin{array}{l}\text { (9158834) YOVANIS } \\
\text { HERRERA PALOMINO }\end{array}$ & 1,52 \\
\hline 14 & $15 / 05 / 2008$ & $\begin{array}{c}\text { DEMÁS CONCESIBLES, GRAVA, } \\
\text { ARENA, ZAHORRA }\end{array}$ & OTROS & $\begin{array}{l}\text { (933387) SAMUEL } \\
\text { CASTILLA PASO }\end{array}$ & 0,92 \\
\hline 15 & $04 / 06 / 2010$ & $\begin{array}{c}\text { MATERIALES DE CONSTRUCCIÓN, } \\
\text { DEMÁS CONCESIBLES }\end{array}$ & $\begin{array}{l}\text { MATERIALES DE } \\
\text { CONSTRUCCIÓN }\end{array}$ & $\begin{array}{l}\text { (9110876) LUIS EDMUNDO } \\
\text { MARTELO YEPES }\end{array}$ & 32,80 \\
\hline 16 & $01 / 09 / 2009$ & $\begin{array}{c}\text { DEMÁS CONCESIBLES, } \\
\text { MATERIALES DE CONSTRUCCIÓN }\end{array}$ & $\begin{array}{l}\text { MATERIALES DE } \\
\text { CONSTRUCCIÓN }\end{array}$ & $\begin{array}{l}\text { (6810002) EMIRO DE JESÚS } \\
\text { PÉREZ VERGARA }\end{array}$ & 8,07 \\
\hline 17 & $09 / 09 / 2009$ & MATERIALES DE CONSTRUCCIÓN & $\begin{array}{l}\text { MATERIALES DE } \\
\text { CONSTRUCCIÓN }\end{array}$ & $\begin{array}{c}\text { (9001351683) AUTOPISTAS } \\
\text { DE LA SABANA SA }\end{array}$ & 154,40 \\
\hline 18 & $17 / 05 / 1994$ & DEMÁS CONCESIBLES, PUZOLANA & OTROS & $\begin{array}{c}\text { (8901002510) CEMENTOS } \\
\text { ARGOS S.A }\end{array}$ & 601,00 \\
\hline 19 & $31 / 07 / 1996$ & CHERT, SILICEOS & OTROS & $\begin{array}{c}\text { (8901002510) CEMENTOS } \\
\text { ARGOS S.A }\end{array}$ & 160,00 \\
\hline 20 & $16 / 02 / 2010$ & $\begin{array}{c}\text { DOLOMITA, ARCILLA, CALIZA, } \\
\text { GRAVA, ARENA }\end{array}$ & OTROS & $\begin{array}{l}\text { (33166022) MARÍA DEL } \\
\text { CARMEN FADUL CHADID }\end{array}$ & 109,71 \\
\hline 21 & $05 / 02 / 1997$ & CHERT, DEMÁS CONCESIBLES & OTROS & $\begin{array}{c}\text { (8901002510) CEMENTOS } \\
\text { ARGOS S.A }\end{array}$ & 69,65 \\
\hline 22 & $04 / 05 / 1993$ & $\begin{array}{l}\text { CHERT, DEMÁS CONCESIBLES, } \\
\text { MATERIALES DE CONSTRUCCIÓN }\end{array}$ & $\begin{array}{l}\text { MATERIALES DE } \\
\text { CONSTRUCCIÓN }\end{array}$ & $\begin{array}{c}\text { (8901002510) CEMENTOS } \\
\text { ARGOS S.A }\end{array}$ & 126,50 \\
\hline 23 & $25 / 02 / 2010$ & MATERIALES DE CONSTRUCCIÓN & $\begin{array}{l}\text { MATERIALES DE } \\
\text { CONSTRUCCIÓN }\end{array}$ & $\begin{array}{l}\text { (92531678) FERNANDO } \\
\text { ROMERO ROMERO }\end{array}$ & 10,94 \\
\hline 24 & $27 / 10 / 2009$ & MATERIALES DE CONSTRUCCIÓN & $\begin{array}{l}\text { MATERIALES DE } \\
\text { CONSTRUCCIÓN }\end{array}$ & $\begin{array}{l}\text { (8001306324) MINISTERIO } \\
\text { DE DEFENSA NACIONAL - } \\
\text { EJERCITO NACIONAL }\end{array}$ & 5,71 \\
\hline
\end{tabular}




\begin{tabular}{|c|c|c|c|c|}
\hline $28 / 10 / 2009$ & MATERIALES DE CONSTRUCCIÓN & $\begin{array}{l}\text { MATERIALES DE } \\
\text { CONSTRUCCIÓN }\end{array}$ & $\begin{array}{l}\text { (8001306324) MINISTERIO } \\
\text { DE DEFENSA NACIONAL - } \\
\text { EJERCITO NACIONAL }\end{array}$ & 99,02 \\
\hline $28 / 04 / 2010$ & MATERIALES DE CONSTRUCCIÓN & $\begin{array}{l}\text { MATERIALES DE } \\
\text { CONSTRUCCIÓN }\end{array}$ & $\begin{array}{l}\text { (92276381) ELIEVER } \\
\text { ANTONIO DE HOYOS ÁVILA }\end{array}$ & 53,29 \\
\hline $13 / 05 / 2010$ & MATERIALES DE CONSTRUCCIÓN & $\begin{array}{l}\text { MATERIALES DE } \\
\text { CONSTRUCCIÓN }\end{array}$ & $\begin{array}{c}\text { (92275274) PABLO } \\
\text { ALEJANDRO ÁLVAREZ ALVIZ }\end{array}$ & 11,07 \\
\hline $30 / 06 / 2010$ & $\begin{array}{c}\text { ARENAS Y GRAVAS SILÍCEAS } \\
\text { ELABORADAS (TRITURADAS, MOLIDAS } \\
\text { O PULVERIZADAS), MATERIALES } \\
\text { DE CONSTRUCCIÓN, OTROS } \\
\text { MINERALES NCP }\end{array}$ & $\begin{array}{l}\text { MATERIALES DE } \\
\text { CONSTRUCCIÓN }\end{array}$ & $\begin{array}{l}\text { (19191886) PABLO } \\
\text { EDUARDO BULLA DUEÑAS }\end{array}$ & 88,89 \\
\hline $23 / 05 / 2012$ & $\begin{array}{c}\text { ARENAS Y GRAVAS NATURALES } \\
\text { Y SILICEAS, MATERIALES DE } \\
\text { CONSTRUCCIÓN, DEMÁS CONCESIBLES }\end{array}$ & $\begin{array}{l}\text { MATERIALES DE } \\
\text { CONSTRUCCIÓN }\end{array}$ & $\begin{array}{l}\text { (9002084604) ASOCIACIÓN } \\
\text { DE ARENEROS DEL PIÑAL }\end{array}$ & 45,45 \\
\hline $05 / 04 / 2011$ & $\begin{array}{l}\text { MATERIALES DE CONSTRUCCIÓN, } \\
\text { DEMÁS CONCESIBLES }\end{array}$ & $\begin{array}{l}\text { MATERIALES DE } \\
\text { CONSTRUCCIÓN }\end{array}$ & $\begin{array}{l}\text { (3501744) CRISTOBAL DÍAZ } \\
\text { GONZÁLEZ, (9000467451) } \\
\text { MELODIAZ LTDA }\end{array}$ & 103,00 \\
\hline $05 / 03 / 2013$ & $\begin{array}{l}\text { MATERIALES DE CONSTRUCCIÓN, } \\
\text { DEMÁS CONCESIBLES }\end{array}$ & $\begin{array}{l}\text { MATERIALES DE } \\
\text { CONSTRUCCIÓN }\end{array}$ & $\begin{array}{l}\text { (45691007) JESSICA MELINA } \\
\text { FERNÁNDEZ ADUEN }\end{array}$ & 99,64 \\
\hline $12 / 03 / 2013$ & MATERIALES DE CONSTRUCCIÓN & $\begin{array}{l}\text { MATERIALES DE } \\
\text { CONSTRUCCIÓN }\end{array}$ & $\begin{array}{l}\text { (800100729) MUNICIPIO } \\
\text { DE OVEJAS }\end{array}$ & 40,42 \\
\hline $22 / 09 / 2010$ & $\begin{array}{l}\text { MATERIALES DE CONSTRUCCIÓN, } \\
\text { DEMÁS CONCESIBLES }\end{array}$ & $\begin{array}{l}\text { MATERIALES DE } \\
\text { CONSTRUCCIÓN }\end{array}$ & $\begin{array}{c}(9000467451) \\
\text { MELODIAZ LTDA }\end{array}$ & 521,69 \\
\hline $06 / 05 / 2013$ & $\begin{array}{l}\text { ARENAS Y GRAVAS } \\
\text { NATURALES Y SILICEAS }\end{array}$ & OTROS & $\begin{array}{l}(9002100163) \\
\text { ASOCIACIÓN DE MINEROS } \\
\text { DE TOLUVIEJODE } \\
\text { LA EXPLORACIÓN Y } \\
\text { EXPLOTACIÓN DE ARENA Y } \\
\text { OTROS MINERALES }\end{array}$ & 32,61 \\
\hline $17 / 12 / 2010$ & $\begin{array}{c}\text { ARENAS Y GRAVAS NATURALES } \\
\text { Y SILICEAS, GRAVAS NATURALES, } \\
\text { MATERIALES DE CONSTRUCCIÓN, } \\
\text { DEMÁS CONCESIBLES }\end{array}$ & $\begin{array}{l}\text { MATERIALES DE } \\
\text { CONSTRUCCIÓN }\end{array}$ & $\begin{array}{c}\text { (9292278) GUSTAVO JOSE } \\
\text { GONZALEZ OROZCO, } \\
\text { (23200504) CARMEN EDITH } \\
\text { JARABA SERPA }\end{array}$ & 140,08 \\
\hline $16 / 04 / 2013$ & MATERIALES DE CONSTRUCCIÓN & $\begin{array}{l}\text { MATERIALES DE } \\
\text { CONSTRUCCIÓN }\end{array}$ & $\begin{array}{l}\text { (9003730922) YUMA } \\
\text { CONCESIONARIA S.A. }\end{array}$ & 32,28 \\
\hline $19 / 10 / 2010$ & $\begin{array}{l}\text { MATERIALES DE CONSTRUCCIÓN, } \\
\text { DEMÁS CONCESIBLES }\end{array}$ & $\begin{array}{l}\text { MATERIALES DE } \\
\text { CONSTRUCCIÓN }\end{array}$ & $\begin{array}{l}\text { (5712856) OMAR WILLIAM } \\
\text { CAMACHO GAMBA }\end{array}$ & 296,89 \\
\hline $13 / 03 / 2012$ & MATERIALES DE CONSTRUCCIÓN & $\begin{array}{l}\text { MATERIALES DE } \\
\text { CONSTRUCCIÓN }\end{array}$ & $\begin{array}{l}\text { (8922005923) MUNICIPIO } \\
\text { DE SAN ONOFRE }\end{array}$ & 4,70 \\
\hline $07 / 10 / 2008$ & CARBÓN, DEMÁS CONCESIBLES & CARBÓN & $\begin{array}{l}(8000248610) \\
\text { GEOCOSTA LTDA }\end{array}$ & $1.237,00$ \\
\hline $01 / 02 / 2010$ & CARBÓN, DEMÁS CONCESIBLES & CARBÓN & $\begin{array}{l}\text { (8901072616) JUAN } \\
\text { MANUEL RUISECO V. } \\
\text { Y CIA. S.C.A }\end{array}$ & $1.999,32$ \\
\hline $01 / 02 / 2010$ & CARBÓN, DEMÁS CONCESIBLES & CARBÓN & $\begin{array}{c}\text { (8305092946) GRUPO } \\
\text { PENTA S.A }\end{array}$ & $1.999,80$ \\
\hline $31 / 01 / 1997$ & CALIZA & CALIZA & $\begin{array}{l}(8922005812) \\
\text { AGROINDUSTRIAS } \\
\text { DEL CARIBE S.A. }\end{array}$ & 21,08 \\
\hline $13 / 06 / 1990$ & MÁRMOL, CALIZA & CALIZA & $\begin{array}{l}\text { (7777700164) COLOMBIANA } \\
\text { DE PIEDRAS LTDA. }\end{array}$ & 24,00 \\
\hline $07 / 02 / 2005$ & DEMÁS CONCESIBLES, CALIZA & CALIZA & $\begin{array}{l}\text { (6818392) RICARDO } \\
\text { FERIS CHADID }\end{array}$ & 43,32 \\
\hline $15 / 05 / 1990$ & CALIZA & CALIZA & $\begin{array}{l}\text { (6818392) RICARDO } \\
\text { FERIS CHADID }\end{array}$ & 65,54 \\
\hline $27 / 02 / 2009$ & $\begin{array}{l}\text { ROCA O PIEDRA CALIZA EN BRUTO, } \\
\text { DEMÁS CONCESIBLES, MÁRMOL }\end{array}$ & CALIZA & $\begin{array}{l}\text { (72218652) ROBERTO JOSE } \\
\text { GUTIERREZ DANÍES }\end{array}$ & 414,68 \\
\hline
\end{tabular}




\begin{tabular}{|c|c|c|c|c|c|}
\hline 47 & $27 / 08 / 1990$ & CALIZA & CALIZA & $\begin{array}{c}\text { (8901002510) CEMENTOS } \\
\text { ARGOS S.A }\end{array}$ & 987,47 \\
\hline 48 & 28/01/1991 & DEMÁS CONCESIBLES, CALIZA & CALIZA & $\begin{array}{l}\text { (6818392) RICARDO } \\
\text { FERIS CHADID }\end{array}$ & 97,91 \\
\hline 49 & $24 / 05 / 2007$ & $\begin{array}{l}\text { ASOCIADOS, ARCILLA, } \\
\text { CALIZA, MARGAS }\end{array}$ & CALIZA & $\begin{array}{c}\text { (8901002510) CEMENTOS } \\
\text { ARGOS S.A }\end{array}$ & 391,00 \\
\hline 50 & $10 / 09 / 1991$ & CALIZA & CALIZA & $\begin{array}{l}\text { (6818392) RICARDO } \\
\text { FERIS CHADID }\end{array}$ & 56,36 \\
\hline 51 & $26 / 09 / 2008$ & DEMÁS CONCESIBLES, CALIZA & CALIZA & $\begin{array}{c}(9001431014) \\
\text { EXPLOMINERALES } \\
\text { DEL NORTE SA }\end{array}$ & $1.189,0$ \\
\hline 52 & $29 / 04 / 2008$ & DEMÁS CONCESIBLES, CALIZA & CALIZA & $\begin{array}{l}(9001431014) \\
\text { EXPLOMINERALES } \\
\text { DEL NORTE SA }\end{array}$ & $1.526,87$ \\
\hline 53 & $24 / 07 / 2007$ & ARCILLA, CALIZA & CALIZA & $\begin{array}{c}\text { (73227168) CARLOS } \\
\text { ALBERTO VÁSQUEZ DE ORO }\end{array}$ & 9,88 \\
\hline 54 & $11 / 06 / 2008$ & DEMÁS CONCESIBLES, CALIZA & CALIZA & $\begin{array}{c}(9001431014) \\
\text { EXPLOMINERALES } \\
\text { DEL NORTE SA }\end{array}$ & $1.841,5$ \\
\hline 55 & $27 / 12 / 2005$ & $\begin{array}{l}\text { ASOCIADOS, ARCILLA, } \\
\text { CALIZA, MARGAS }\end{array}$ & CALIZA & $\begin{array}{c}\text { (8901002510) CEMENTOS } \\
\text { ARGOS S.A }\end{array}$ & 494,50 \\
\hline 56 & $16 / 07 / 2009$ & DEMÁS CONCESIBLES, CALIZA & CALIZA & $\begin{array}{l}\text { (8305092946) GRUPO } \\
\text { PENTA S.A }\end{array}$ & $1.701,2^{2}$ \\
\hline 57 & $16 / 07 / 2009$ & $\begin{array}{l}\text { ROCA O PIEDRA CALIZA EN BRUTO, } \\
\text { CARBÓN, DEMÁS CONCESIBLES }\end{array}$ & CALIZA & $\begin{array}{l}\text { (8305092946) GRUPO } \\
\text { PENTA S.A } \\
\end{array}$ & $1.923,72$ \\
\hline 58 & $18 / 12 / 2009$ & $\begin{array}{c}\text { ROCA O PIEDRA CALIZA EN BRUTO, } \\
\text { DEMÁS CONCESIBLES }\end{array}$ & CALIZA & $\begin{array}{l}\text { (6810001) JORGE ENRIQUE } \\
\text { MENDOZA VERGARA }\end{array}$ & 69,23 \\
\hline 59 & $11 / 02 / 1994$ & CALIZA & CALIZA & $\begin{array}{l}\text { (8922005812) } \\
\text { AGROINDUSTRIAS } \\
\text { DEL CARIBE S.A. }\end{array}$ & 82,08 \\
\hline 60 & $12 / 08 / 2009$ & DEMÁS CONCESIBLES, CALIZA & CALIZA & $\begin{array}{c}\text { (8305148533) } \\
\text { CARBOMINE S.A.S. }\end{array}$ & $1.004,2 C$ \\
\hline 61 & $29 / 09 / 2009$ & ARCILLA, CALIZA, ROCAS & CALIZA & $\begin{array}{c}\text { (8901002510) CEMENTOS } \\
\text { ARGOS S.A }\end{array}$ & 382,16 \\
\hline 62 & $11 / 03 / 2010$ & CALIZA TRITURADA O MOLIDA & CALIZA & $\begin{array}{c}\text { (72218652) ROBERTO JOSE } \\
\text { GUTIERREZ DANÍES }\end{array}$ & 2,51 \\
\hline 63 & $31 / 03 / 1997$ & CALIZA & CALIZA & $\begin{array}{c}\text { (8230038581) COOPERATIVA } \\
\text { DE PROCESADORES } \\
\text { DE PIEDRA CALIZA DE } \\
\text { VARSOVIA Y GUALÓN } \\
\text { COOMULPROPICAL }\end{array}$ & 15,28 \\
\hline 64 & $07 / 10 / 2005$ & DEMÁS CONCESIBLES, CALIZA & CALIZA & $\begin{array}{l}\text { (64551725) MARÍA SALIMA } \\
\text { GUERRA DE LA ESPRIELLA }\end{array}$ & 7,80 \\
\hline 65 & $11 / 10 / 2005$ & DEMÁS CONCESIBLES, CALIZA & CALIZA & $\begin{array}{l}\text { (64551725) MARÍA SALIMA } \\
\text { GUERRA DE LA ESPRIELLA }\end{array}$ & 963,13 \\
\hline 66 & $09 / 11 / 2010$ & $\begin{array}{l}\text { CALIZA TRITURADA O MOLIDA, } \\
\text { DEMÁS CONCESIBLES }\end{array}$ & CALIZA & $\begin{array}{l}\text { (72218652) ROBERTO JOSE } \\
\text { GUTIERREZ DANÍES }\end{array}$ & 0,61 \\
\hline 67 & $25 / 01 / 2012$ & $\begin{array}{c}\text { CALIZA TRITURADA O MOLIDA, } \\
\text { MATERIALES DE CONSTRUCCIÓN, } \\
\text { DEMÁS CONCESIBLES }\end{array}$ & CALIZA & $\begin{array}{l}\text { (92540811) DANIEL FELIPE } \\
\text { MERLANO PORRAS }\end{array}$ & 8,55 \\
\hline 68 & $24 / 05 / 2007$ & ARCILLA, CALIZA, MARGAS & CALIZA & $\begin{array}{c}\text { (8901002510) CEMENTOS } \\
\text { ARGOS S.A }\end{array}$ & 463,15 \\
\hline
\end{tabular}

